\title{
CORRESPONDENCE
}

\section{THE GIANT AWAKES}

\author{
Valerĭ I. Murakhveri \\ Vvedenskogo ul. 13-2-288 \\ 117342 Moscow \\ USSR
}

\begin{abstract}
"Much water passed under the bridge since the first Soviet chess program was successful in the international arena. We, of course, refer to Kaissa, which ran on an IBM/360 - compatible computer. Due to many reasons, a deep stagnation followed in this field too. However, not all traces of activity were extinct so that when some PCcompatibles were no longer a rarity in our country, a number of chess programs appeared. Also, an electronic manufacturer had developed a chess microcomputer which, seeing it was a first attempt, was creditable. The second USSR tournament for chess programs (Moscow, April 4-6, 1989) had six entries, 5 of them for MS/DOS and the one, a 16K micro, Elektronica, the chess computer referred to.

The general level was rather mediocre, showing bad blunders and a very poor endgame technique. The final round of the all-play-all tournament decided the race: the Algir program by A. Pokrovsky which ran on an IBM PC/AT. The winning program has been written in Assembler resulting in a load module of $80 \mathrm{~K}$ and requiring some $140 \mathrm{~K}$ of memory. It uses black-and-white graphics, emits sounds and has an attractive presentation. In the final decisive game which we present, Elektronica was forced to change its playing level downwards by move 30 since it would be in time trouble otherwise. This goes a long way towards explaining what happened. The final outcome was for Algir 4.5 points out of 5, Elektronica gaining 4 points.
\end{abstract}

ELEKTRONIKA - ALGIR. 1. Nf3 d5 2. d4 e6 3. c4 Nc6 4. cxd5 exd5 5. Nc3 Nf6 6. Qa4 Bb4 7. a3 Ba5 8. b4 Bb6 9. Bg5 0-0 10. Bxf6 gxf6 11. e3 Ne7 12. Bd3 Bf5 13. Bxf5 Nxf5 14. 0-0 Ne7 15. Qc2 Re8 16. a4 a6 17. a5 Ba7 18. Rac1 Rc8 19. b5 c6 20. bxa6 bxa6 21. Qa4 Rb8 22. Rb1 f5 23. Ne5 c5 24. Nd7 Rxb1 25. Nxb1 Kh8 26. dxc5 Rg8 27. Ne5 Rg7 28. c6 Qc7 29. Qa1 Nxc6 30. Nxc6 Qxc6 31. Qe5 d4 32. g3 dxe3 33. fxe3 Qe4 34. Qxe4 fxe4 35. Kf2 f5 36. Nc3 Rc7 37. Nd5 Rc5 38. Rd1 Rxa5 39. Nc3 Bc5 40. Rd7 Ra3 41. Nd5 Ra2+ 42. Kg1 Rd2 43. Rd8+ Kg7 44. Rd7+ Kh6 45. h3 a5 46. g4 f4 47. g5+ Kxg5 48. h4+ Kxh4 49. Rxh7+ Kg5 50. Rg7+ Kh6 51. Rd7 a4 52. Kf1 fxe3 53. Ke1 a3 54. Nf6 Rxd7 55. Nxd7 a2 56. Nxc5 a1=Q+ 57. Ke2 Qd4 58. Nxe4 Qxe4 59. Kd1 Qd3+ 60. Kc1 e2 61. Kb2 e1=Q 62. Ka2 Qb4 63. Ka1 Qa6 mate (0-1)."

\section{HISTORY OF THE "SAFETY" ALGORITHM}

\author{
Dan Heisman \\ 102 Chesapeak Dr. \\ Newton, PA, 18940 / USA
}

"I was quite interested in the historical derivation of the "safety" algorithm that was stated as "interesting but as yet untested..." by the "SEX Algorithm" authors Levy, Broughton and Taylor (ICCA Journal, Vol. 12, No. 1, p. 17).

This algorithm, which involves the relative safety of computer moves, has been proposed by myself over 14 years ago. Around 1975 I made a special trip to Carnegie-Mellon, where I had special meetings with Berliner and, I believe, Newell. I proposed this algorithm (in terms of some deltas and epsilons), and it was met with some interest. Newell said that, although I had come up with the idea independently, it was similar to an idea of Slater's and he recommended one of the latter's books, which I purchased. Berliner gave me a copy of his thesis. Since 